\title{
Highly structured wind in Vela X-1
}

\section{Ingo Kreykenbohm ${ }^{* 1,2}$, Jörn Wilms ${ }^{1,2}$, Peter Kretschmar ${ }^{3}$, José Miguel Torrejón ${ }^{4,5}$, Katja Pottschmidt ${ }^{6,7}$, Manfred Hanke ${ }^{1,2}$, Andrea Santangelo ${ }^{8}$, Carlo Ferrigno ${ }^{8,9,10}$, and Rüdiger Staubert ${ }^{8}$}

${ }^{1}$ Dr. Karl Remeis-Sternwarte Bamberg, Sternwartstrasse 7, 96049 Bamberg, Germany,

${ }^{2}$ Erlangen Centre for Astroparticle Physics, Erwin-Rommel-Str. 1, 91058 Erlangen, Germany

${ }^{3}$ European Space Astronomy Centre, Villafranca del Castillo, P.O. Box 78, 28691 Villanueva de la Cañada, Madrid, Spain

${ }^{4}$ Departamento de Física, Ingeniería de Sistemas y Teoría de la Señal, Escuela Politénica Superior, Universidad de Alicante, Ap. 99, 03080 Alicante, Spain

${ }^{5}$ Kavli Institute for Astrophysics and Space Research, Massachusetts Institute of Technology, Cambridge, MA 02139, USA

${ }^{6}$ CRESST, University of Maryland, Baltimore County, 1000 Hilltop Circle, Baltimore, MD 21250, USA

${ }^{7}$ NASA Goddard Space Flight Center, Astrophysics Science Division, Code 661, Greenbelt, MD 20771, USA

${ }^{8}$ Kepler Center for Astro and Particle Physics, Sand 1, 72076 Tübingen, Germany

${ }^{9}$ INTEGRAL Science Data Centre, 16 ch. d'Écogia, 1290 Versoix, Switzerland

${ }^{10}$ IASF-INAF, via Ugo la Malfa 153, 90136 Palermo, Italy

E-mail:

ingo.kreykenbohmesternwarte.uni-erlangen.de

We present an in-depth analysis of the spectral and temporal behavior of a long almost uninterrupted INTEGRAL observation of Vela X-1 in Nov/Dec 2003. In addition to an already high activity level, Vela X-1 exhibited several very intense flares with a maximum intensity of more than $5 \mathrm{Crab}$ in the $20-40 \mathrm{keV}$ band. Furthermore Vela X-1 exhibited several off states where the source became undetectable with ISGRI. We interpret flares and off states as being due to the strongly structured wind of the optical companion: when Vela X-1 encounters a cavity in the wind with strongly reduced density, the flux drops, thus potentially triggering the onset of the propeller effect which inhibits further accretion, thus giving rise to the off states. The required drop in density to trigger the propeller effect in Vela X-1 is of the same order as predicted by theoretical papers for the densities in the $\mathrm{OB}$ star winds. The same structured wind can give rise to the giant flares when Vela X-1 encounters a dense blob in the wind. Further temporal analysis reveals that a short lived QPO with a period of $6800 \mathrm{sec}$ is present. The part of the light curve during which the QPO is present is very close to the off states and just following a high intensity state, thus showing that all these phenomena are related.

7th INTEGRAL Workshop - An INTEGRAL View of Compact Objects

September 8-11 2008

Copenhagen, Denmark

\footnotetext{
* Speaker.
} 


\section{Introduction}

Vela X-1 is a high mass X-ray binary (HMXB) consisting of the super giant HD 77581 and a massive $\left(1.9 M_{\odot}\right.$ [1]) neutron star in a 8.964 day orbit [2]. The optical companion has a mass of $\sim 23 M_{\odot}$ and a radius of $\sim 30 R_{\odot}$ [2]. The neutron star is deeply embedded in the dense stellar wind of the donor HD $77581\left(\dot{M}_{\star}=4 \times 10^{-6} M_{\odot} \mathrm{yr}^{-1}\right)$ [3]. X-ray lines indicate that this wind is inhomogeneous with many dense clumps [4] embedded in a far thinner, highly ionized component [5].

The neutron star has a long spin period of $\sim 283 \mathrm{~s}$ [6]. The evolution of the spin period is best described by a random walk as expected for a wind-accreting system [7]. Although the source exhibits strong pulse-to-pulse variations, a pulse-profile folded over several pulse periods shows remarkable stability [8], even over decades [9]. At energies below $5 \mathrm{keV}$, the pulse-profile consists of a complex five-peaked structure, which transforms at energies above $20 \mathrm{keV}$ into a simple doublepeaked pulse-profile [8] where the two peaks are thought to be due to the two accreting magnetic poles of the neutron star.

With an X-ray luminosity of $\sim 4 \times 10^{36} \mathrm{erg} \mathrm{s}^{-1}$, Vela X-1 is a typical high mass X-ray binary. Previous observations have shown that the source is strongly variable with reductions to less than $10 \%$ of its normal value $[10,11,12,13]$, while periods of increased activity have also been observed during which the flux increases within an hour to a multiple of the previous value, reaching peak flux levels close to $1 \mathrm{Crab}[11,14,15]$. In this respect, Vela X-1 is similar to sources such as 4U 1700-377 and 4U 1907+09, for which low luminosity states and flares have also been observed, as is rather typical for wind-accreting systems $[16,17,18,19]$. Although Vela X-1 is a well studied object, only observations by INTEGRAL revealed that the flares in Vela X-1 can be brighter than previously anticipated $[10,20,21]$.

\section{Data}

INTEGRAL observed the Vela region continuously for five consecutive INTEGRAL revolutions from revolution 137 (JD 2452970.86) until revolution 141 (JD 2452985.44) resulting in approximately $1 \mathrm{Msec}$ of data (see Fig. 1).

We used all available science windows ( $\mathrm{ScWs}$ ) to be able to derive a contiguous light curve with as few interruptions as possible. Since Vela X-1 is a bright source, the OSA can detect the source and determine its flux level accurately even when the source is at high off-axis angles.

Apart from Vela X-1, also 4U 0836-429, H 0918-5459, the Vela Pulsar, and two sources first reported by INTEGRAL $[22,23]$ are detected. Vela X-1 is widely separated from the other sources such that contamination of the spectrum of Vela X-1 is of no concern. Data from JEM-X and SPI have not been used in this analysis due to the far smaller field-of-view of JEM-X and since Vela X-1 is off-center in the observed field (Vela X-1 was only within the fully coded field-of-view of JEM-X for less than ten out of the $\sim 550$ individual pointings).

\section{Data analysis}

Vela X-1 was found in a highly variable state during the observation. While Vela X-1 is known to be a variable source $[11,24]$, the behavior found in this observation $[10,20]$ is indeed extreme. 


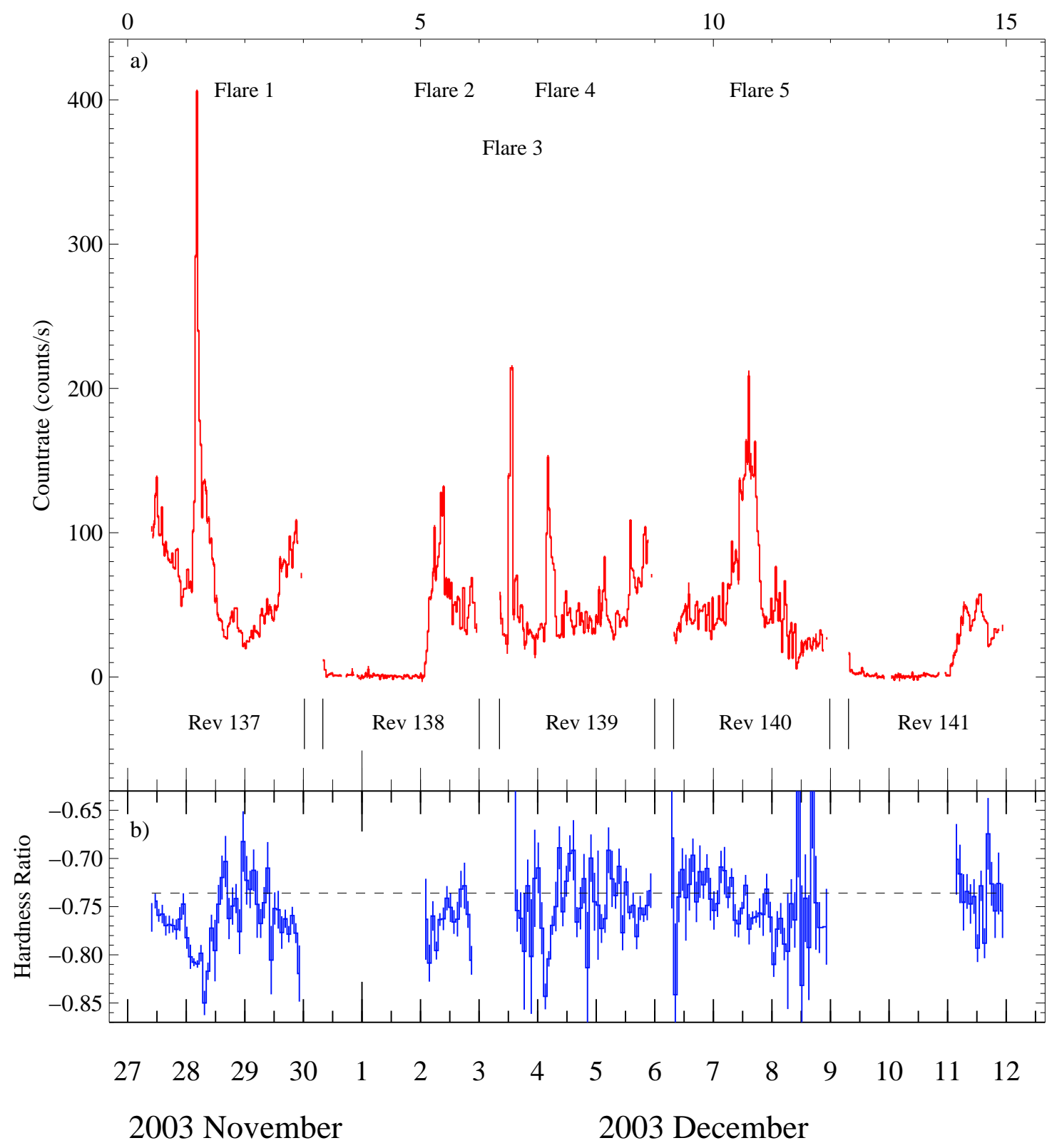

Figure 1: a) ISGRI ScW by ScW light curve in the $20-40 \mathrm{keV}$ energy band and b) hardness ratio. The labels indicate the revolution number (from [10]).

Most importantly, on 2003 November 28 (JD 2452971.67), INTEGRAL observed an extremely bright flare (flare 1; see Fig. 1). During the flare, the $20-40 \mathrm{keV}$ count rate increased from a ScW averaged pre-flare value of $\sim 55$ counts s$^{-1}\left(\sim 300 \mathrm{mCrab}\right.$, or $\left.1.6 \times 10^{-9} \mathrm{erg} \mathrm{cm}^{-2} \mathrm{~s}^{-1}\right)$ by a factor of more than seven to 405 counts s$^{-1}$ ( $\left.2.3 \mathrm{Crab}\right)$ within only 90 minutes.

After the peak, the flare decayed quickly to an intensity level of $<1 \mathrm{Crab}$ and within $\sim 11 \mathrm{~h}$ to a ScW averaged count rate of $\sim 35$ counts s$^{-1}$ ( $200 \mathrm{mCrab}$ ), somewhat lower than before the onset of the flare (see Fig. 1). 


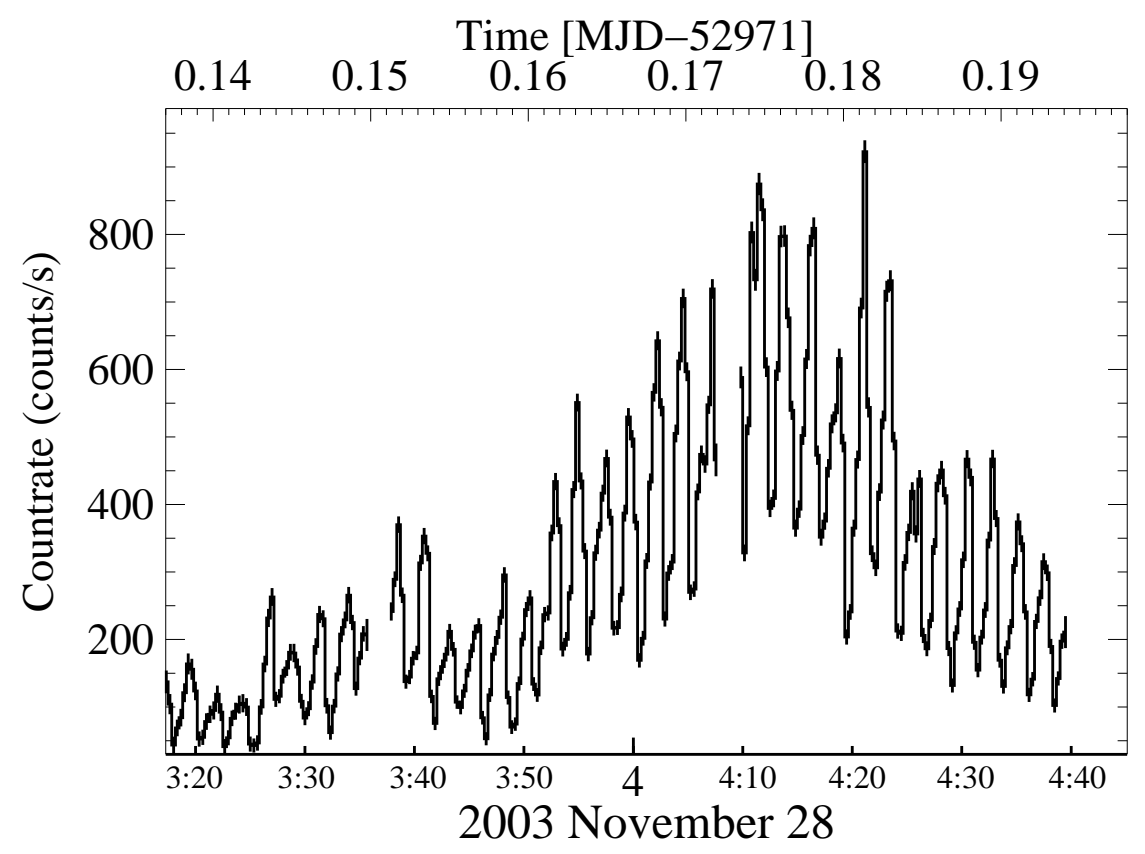

Figure 2: Close up of the light curve of giant flare 1 with a time resolution of $20 \mathrm{~s}$. The peak is reached at MJD 52971.18 with 923 counts s$^{-1}$ (corresponding to $5.2 \mathrm{Crab}$ ) in the 20 to $40 \mathrm{keV}$ band (from [10]).

Table 1: Overview of the observed flares. See Fig. 1 for the numbering of the flares. The time is the onset of the flare. To obtain the peak fluxes $F_{\text {peak }}$, a light curve with a time resolution of $20 \mathrm{~s}$ was used. $T_{\text {rise }}$ is the time from the onset of the flare to the peak, while $T_{\text {total }}$ is the duration of the flare.

\begin{tabular}{rcrrrl}
\hline \hline Flare & $\begin{array}{c}\text { Time } \\
{[\mathrm{MJD}]}\end{array}$ & $\begin{array}{c}\text { Duration } \\
{[\mathrm{s}]}\end{array}$ & \multicolumn{1}{c}{$F_{\text {peak }}$} \\
{$[\mathrm{Crab}]$} & $\frac{T_{\text {rise }}}{T_{\text {total }}}$ & Remarks \\
\hline 1 & 52971.15 & 11200 & 5.2 & 0.15 & giant flare, spectral softening \\
2 & 52975.34 & 5200 & 2.6 & 0.83 & no spectral change \\
3 & 52976.50 & 1800 & 5.3 & 0.28 & giant flare, very short \\
4 & 52977.15 & 12900 & 1.9 & 0.13 & spectral softening \\
5 & 52980.31 & 31400 & 3.9 & 0.63 & high intensity state, no spectral change \\
\hline
\end{tabular}

In the following 13 days, three more flares (flares 2 to 4 , see Table 1 were observed. All three flares were shorter and less intense than flare 1 on a science window averaged basis, but still reached $\mathrm{ScW}$ averaged intensities close to $1 \mathrm{Crab}$.

On 2003 December 7 (JD 245981.10), another intense flare was observed (designated Flare 5, see Fig. 1). Unlike flare 1, during which the brightness of the source increased rapidly, it took $\sim 8 \mathrm{~h}$ for flare 5 to reach its $\mathrm{ScW}$ averaged maximum $20-40 \mathrm{keV}$ flux of $\sim 1.2 \mathrm{Crab}$. The decay lasted $\sim 5 \mathrm{~h}$ until Vela X-1 reached its pre-flare count rate of $\sim 35$ counts s$^{-1}$ (200 mCrab in $20-40 \mathrm{keV}$ ). Although quite bright, flare 5 is therefore significantly less intense than giant flare 1 , and also far longer, i.e. it is a high intensity state.

The analysis of a light curve with a $20 \mathrm{~s}$ time resolution showed that the source reached a peak count rate of $\sim 920$ counts s$^{-1}(5.2 \mathrm{Crab})$ in flare 1 and $\sim 930$ counts s$^{-1}(5.3 \mathrm{Crab})$ in flare 3 . 


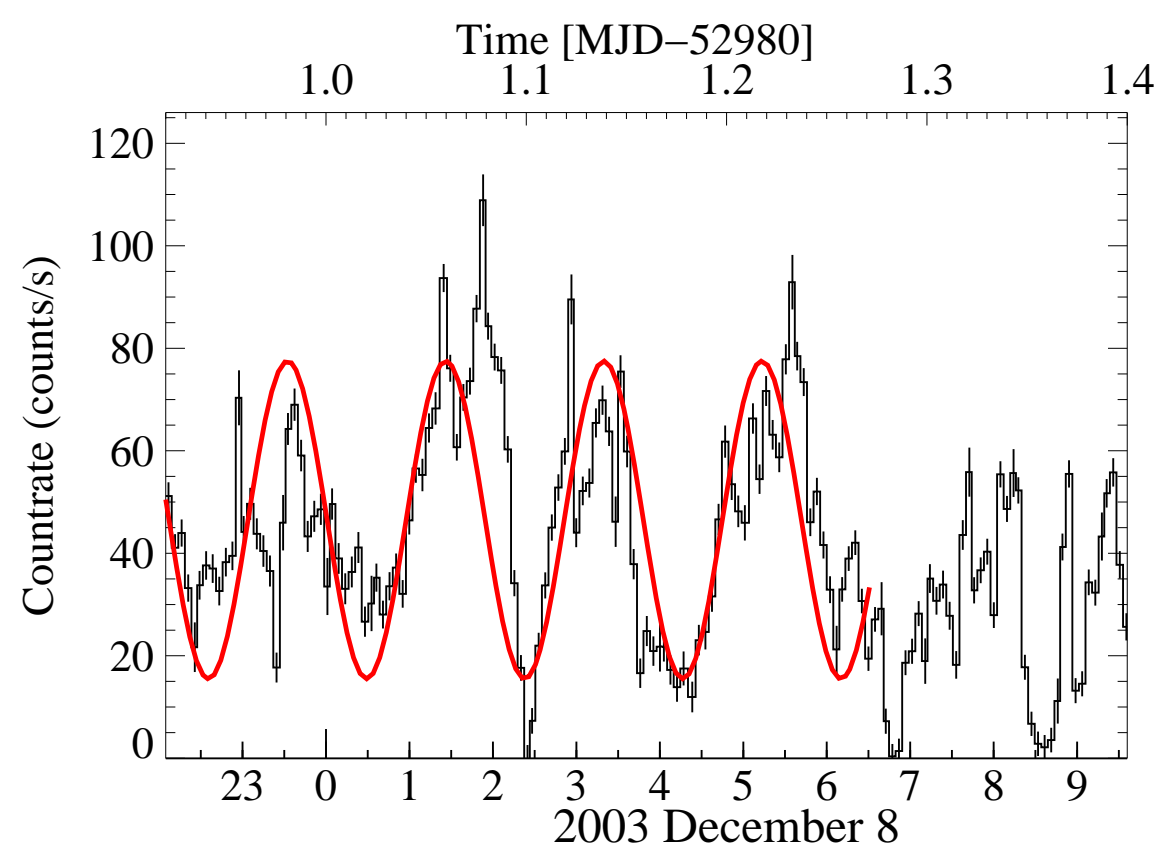

Figure 3: Closeup of the light curve (with a time resolution of $283 \mathrm{~s}$ to remove the pulsations) where the temporary QPO is present. Note that during the trough between $2 \mathrm{~h}$ and $3 \mathrm{~h}$, and especially following the quasi-periodic modulation, the count rate decreased several times to zero for a short time (from [10]).

Flare 3 on December 3 was therefore also a giant flare. However, flare 3 was significantly shorter: the entire flare lasted less than $2000 \mathrm{~s}$, but it was as bright as flare 1 (see Table 1).

Extending the analysis to the non-flaring parts of the light curve, we detected a quasi-periodic oscillation (QPO), similar to other accreting X-ray pulsars. The short-lived QPO with a period of $\sim 6820 \mathrm{sec}$ appears to be quite regular and inconsistent with pure stochastic behavior (see Fig. 3). Subsequent period searches on the corresponding data subset clearly detect the period. We note that the quasi-periodic modulation shown in Fig. 3 is far stronger and inconsistent with the NOMEX effect, which can cause intensity variations from pointing to pointing, but not within a given pointing.

Furthermore, we observed several off states, during which no significant residual flux was detectable by ISGRI (Fig. 4). The onset of these off states occured very suddenly. The luminosity of the source simply drops below the detection limit of ISGRI. At the end of the off states, Vela X-1 switches instantly on again and immediately resumes its normal intensity level. All off states occured within $12 \mathrm{~h}$ from MJD 52981.0 and MJD 52981.5.

\section{Spectral evolution during the flares}

Although the source was extremely bright during the flares, meaningful spectral fits could not be obtained, as the exposure time was to short. We therefore analyze the hardness ratio (see Fig. 1b), which is defined as

$$
\mathrm{HR}=\frac{H-S}{H+S}
$$




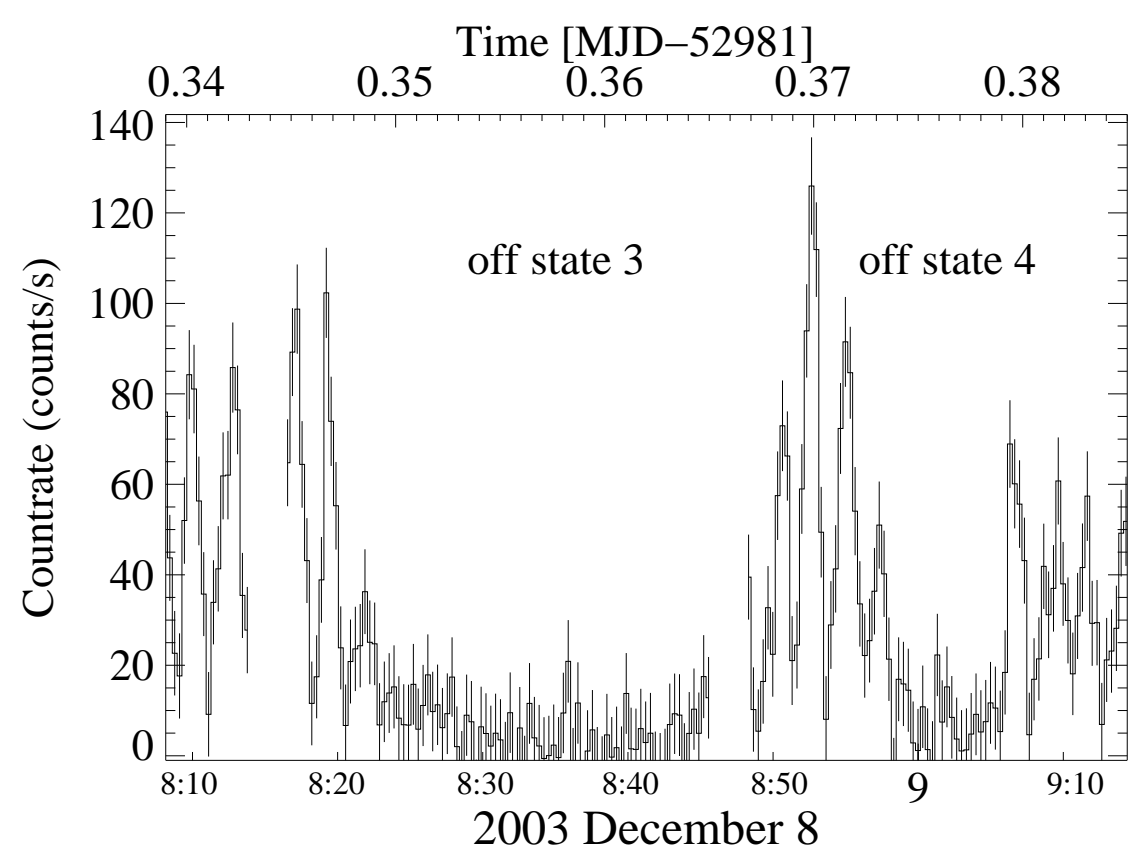

Figure 4: Close-up with a time resolution of $20 \mathrm{sec}$ on off states 3 and 4 of Vela X-1 during which the source becomes undetectable by ISGRI and then turns on again within one hour (from [10]).

where $H$ is the count rate in the hard band $(40-60 \mathrm{keV})$ and $S$ the count rate in the soft band (20-30 keV). While the hardness ratio remained constant throughout most of the observation at $\sim-0.735$, the hardness ratio significantly changed with the onset of flare 1 : it dropped to -0.82 and during the flare to -0.85 (see Fig. 1b). The same for flare 4: the hardness ratio dropped from -0.72 to -0.84 , the same level as in giant flare 1 , although flare 4 was far shorter and reached only a third of the peak flux of flare 1. During flares 2 and 5, however, the hardness ratio did not change.

We then used "Hardness intensity diagrams" (HIDs) to study the spectral evolution of the source (see Fig. 5). Most of the data points are centered around the average values of intensity and hardness ratio. The only exception are the data points from the flares, which are above the general cluster of data points. Due to the spectral softening, the data points of flare 1 and flare 4 are shifted. The softening, however, did not evolve during the flares, but the flares are softer than the average spectrum from the beginning until the end.

\section{Discussion}

\subsection{The flares}

Vela X-1 has always been known to be highly variable with time and to show intensity variations of up to a multiple or a fraction of the original intensity on all time scales. Although Vela X-1 has exhibited extensive flaring activity in the past however, giant flares (as flares 1 and 3) had not been seen before.

The analysis of the hardness ratio shows that there seem to be two types of flares: the first type (flares 1 and 4) shows dramatic increases in the count rate, the onset of the flare is very sudden, 


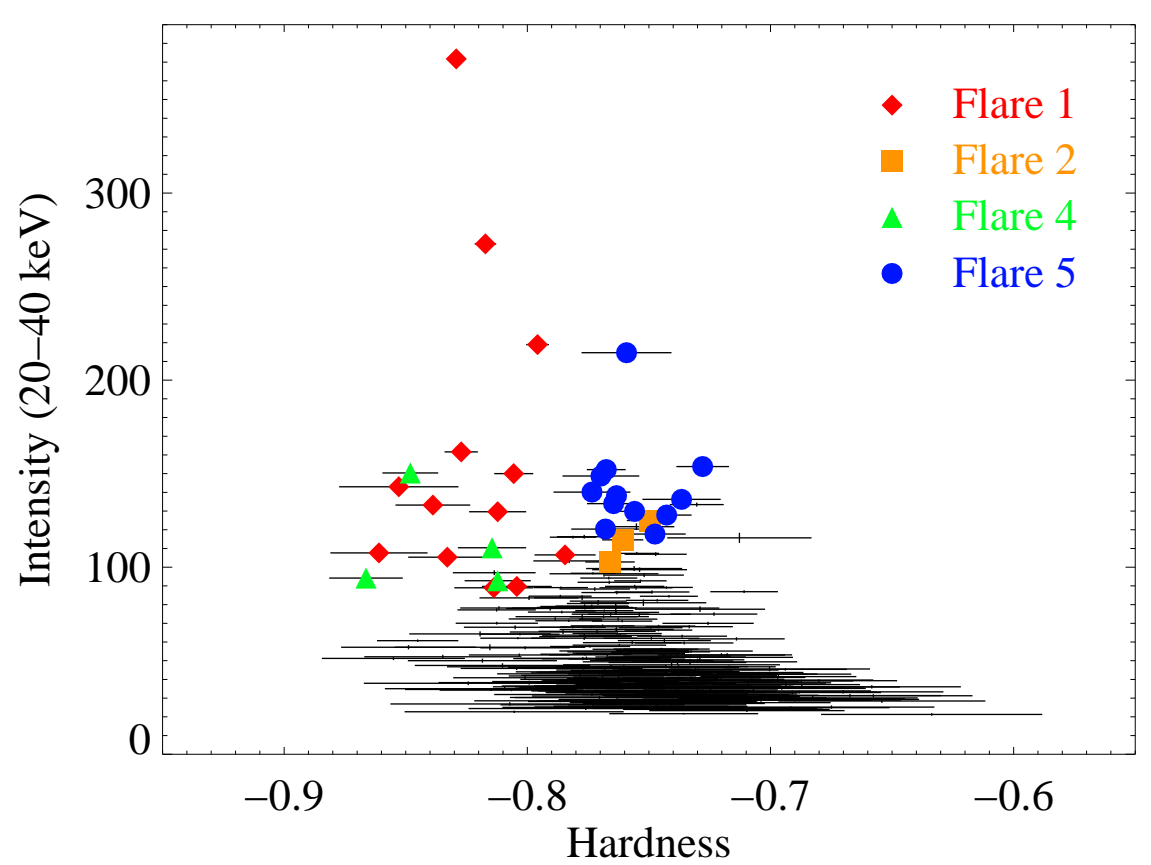

Figure 5: Hardness Intensity Diagram of Vela X-1; data from the eclipses have been excluded. The data points from the flares are indicated by individual symbols. The datapoints from the eclipses have been removed (from [10]).

and the spectrum softens during the flare. The second type is similar to a high intensity state: these flares are longer and the spectrum does not change.

The mechanism behind the flaring activity, however, is not fully understood. It has been shown that a temporary disk may form in Vela X-1 [25]. The disk collapses and the material is accreted onto the neutron star giving rise to a short flare. These predicted flares would last from 15 to 60 minutes, similar to the short flaring activity of Vela X-1. Furthermore, wind accretion is a highly instable process by itself: the accretion wake trailing the neutron star contains filaments which also produce flares when being accreted [26]. The shock trailing the neutron star oscillates creating the "flip-flop instability" which then produces inflows that repeatedly change their direction [27, 28]. The timescale of 45 min matches some of the observed behavior very well, but fails to explain the long flares.

As the local density in a shocked wind varies by a factor of 100 [29], which can explain the flaring X-ray luminosity [4], dense clumps trapped in an otherwise thin and more homogeneous wind might be responsible for long flares [30], when the clumps are being accreted. Such a clump can feed the neutron star with a significantly higher $\dot{M}$ than usual over several hours. In summary, we conclude that the observed long flares are due to a strongly structured OB star wind, while when Vela X-1 is less active, the OB star wind is less structured.

\subsection{The off states}

In a similar way to the flaring activity, the off states $[13,11,10]$ where the source is below the detection limit are remarkable. After the off state observed by RXTE in 1996 [11], the source 
resumed its normal, pulsating behavior without any transition phase. The offstates reported here also occured without a transition phase [10].

The reasons for these off states and the sudden reappearance of pulsations are not understood. So far, several ideas to explain these phenomena have been proposed, however, none can fully explain the observed off states, since all of these ideas require a significantly longer timescale, e.g. clumps in the stellar wind [31] would have to have an unrealistic high optical depth to completely block hard X-rays and can not pass the line of sight in a few seconds to explain the sudden turn on/off behavior of the source. Therefore other mechanisms must be considered.

The wind of OB super giants is inhomogeneous and clumpy $[32,26]$. The density of the stellar wind can vary by several orders of magnitude [33]. Not only clumps, but also holes, i.e. regions of strongly reduced density are present in the wind: there, the density is lower than the average density of the wind by a factor of $10^{3}$ [33]. If the neutron star enters these holes, $\dot{M}$ would then also decrease by a factor of $\sim 10^{3}$ and the X-ray luminosity would be reduced accordingly. Furthermore, the density fluctuations predicted by these models occur suddenly [see Fig. 1 in 33] similar to the onset of the off states (see Fig. 1). If $\dot{M}$ drops due to these density variations in the wind, the Alfvén radius will increase due to the reduced ram pressure of the infalling gas. Once it is larger than the co-rotation radius, accretion onto the neutron star is inhibited, i.e. the X-ray source basically switches off. This scenario is commonly known as the propeller effect [34]. Since the propeller effect depends on the amount of infalling material, the Alfvén radius is not constant. This effect was observed in GX 1+4 [35]: in very low luminosity states, no pulsations were observable, while the source was strongly pulsating in high luminosity states.

Since the strength of the magnetic field of Vela X-1 is known from the observation of the cyclotron lines [36], the critical flux limit for Vela X-1 for the onset of the propeller effect can be obtainted (after [35]): $F_{\mathrm{X} \text {,Propeller,Vela X-1 }} \approx 1.1 \times 10^{-12} \mathrm{erg} \mathrm{cm}^{-2} \mathrm{~s}^{-1}$. Compared with the typical bolometric flux of several times $10^{-9} \mathrm{erg} \mathrm{cm}^{-2} \mathrm{~s}^{-1}$, this critical flux is lower by about three orders of magnitude. This flux limit matches very closely the predicted density variations in the stellar wind of $10^{3-5}$ [32].

We therefore conclude that off states could be caused by a sudden drop in $\dot{M}$ that allows Vela X-1 to enter the propeller regime. Intensity dips, however, are longer, show a smooth transition, and exhibit photoelectric absorption of more than $10^{24} \mathrm{~cm}^{-2}$. These dips are readily explained by a dense blob in the wind passing through the line of sight.

\subsection{Connection with SFXTs}

The similarity between the flares and off states in Vela X-1 and the behavior of Supergiant Fast X-ray Transients (SFXTs) [37] is striking. SFXTs are high mass X-ray binaries that show very brief outbursts on timescales of hours or even only tens of minutes, and then remain undetectable at higher energies for months between outbursts [38]. SFXTs should be rather bright persistent objects [39] since the neutron star is deeply embedded in the dense stellar wind of the optical companion, however, the accretion is inhibited by the propeller effect. It has therefore been proposed that SFXTs harbor a magnetar [40] and that the extremely strong magnetic field $\left(B>10^{15} \mathrm{G}\right)$ effectively inhibts accretion unless the ram pressure of the infalling gas is high enough such that accretion becomes possible for a short time. The giant flares and off states of Vela X-1 are therefore similar to these outbursts: in both cases the accretion of a dense blob of material causes the outburst or 
flare, while reduced material infall causes the source to switch off. In summary, Vela X-1 and SFXTs are rather similar objects, however, SFXTs are usually in the off state, while Vela X-1 is usually in a normal accretion mode.

\section{References}

[1] H. Quaintrell, A. J. Norton, T. D. C. Ash, P. Roche, B. Willems, T. R. Bedding, I. K. Baldry, and R. P. Fender, The mass of the neutron star in Vela X-1 and tidally induced non-radial oscillations in GP Vel, A\&A 401, 313-323 (2003).

[2] M. H. van Kerkwijk, J. van Paradijs, E. J. Zuiderwijk, G. Hammerschlag-Hensberge, L. Kaper, and C. Sterken, Spektroscopy of HD 77581 and the mass of Vela X-1, A\&A 303, 483-496 (1995).

[3] Fumiaki Nagase, Satio Hayakawa, Naohisa Sato, Kuniaki Masai, and Hajime Inoue, Circumstellar matter in the Vela X-1/HD77581 system, PASJ 38, 547-569 (1986).

[4] L. M. Oskinova, W.-R. Hamann, and A. Feldmeier, X-raying clumped stellar winds in proceedings of Clumping in Hot Star Winds, (Potsdam) (Wolf-Rainer Hamann, Achim Feldmeier, and Lidia Oskinova, eds.), 2007, p. 203.

[5] Masao Sako, Duane A. Liedahl, Steven M. Kahn, and Frits Paerels, The X-ray spectrum and global structure of the stellar wind in Vela X-1, ApJ 525, 921-934 (1999).

[6] S. Rappaport and J. E. McClintock, Discovery of a 283 second period in Vela X-1, IAU Circ. 2794 (1975).

[7] J. Ziółkowski, Rotational status of X-ray pulsars, Acta Astronomica 35, 185-198 (1985).

[8] R. Staubert, E. Kendziorra, W. Pietsch, J. Trümper C. Reppin, and W. Voges, Hard X-ray pulses from 4U0900-40, ApJ 239, 1010-1016 (1980).

[9] B. C. Raubenheimer, The pulsed light curve of Vela X-1, A\&A 234, 172 (1990).

[10] I. Kreykenbohm, J. Wilms, P. Kretschmar, J. M. Torrejón, K. Pottschmidt, M. Hanke, A. Santangelo, C. Ferrigno, and R. Staubert, High variability in Vela X-1: giant flares and off states, A\&A 492, 511-525 (2008).

[11] I. Kreykenbohm, P. Kretschmar, J. Wilms, R. Staubert, E. Kendziorra, D. E. Gruber, W. A. Heindl, and R. E. Rothschild, Vela X-1 as seen by RXTE, A\&A 341, 141-150 (1999).

[12] P. Kretschmar, I. Kreykenbohm, J. Wilms, R. Staubert, W. A. Heindl, D. E. Gruber, and R. E. Rothschild, Disappearing pulses in Vela X-1 in proceedings of Proc. 5th Compton Symposium, (M. McConnell and J. Ryan, eds.), AIP Conf. Proc., no. 510, 1999, p. 163.

[13] Hajime Inoue, Yoshiaki Ogawara, Takaya Ohashi, and Izumi Waki, Sudden disappearance of Vela X-1 pulses, PASJ 36, 709-713 (1984). 
[14] F. Haberl and N. E. White, The X-ray absorption spectrum of Vela X-1, ApJ 361, 225-234 (1990).

[15] E. Kendziorra, B. Mony, M. Maisack, R. Staubert, S. Döbereiner, J. Englhauser, W. Pietsch, C. Reppin, J. Trümper, V. Efremov, S. Kaniovsky, Kuznetsov, and R. Sunyaev, Hard X-ray pulses from Vela X-1 in proceedings of Proc. 23rd ESLAB Symp. on Two-Topics in X-Ray Astronomy, (Bologna), ESA SP-296, November 1989, pp. 467-471.

[16] S. Fritz, I. Kreykenbohm, J. Wilms, R. Staubert, F. Bayazit, K. Pottschmidt, J. Rodriguez, and A. Santangelo, A torque reversal of $4 U$ 1907+09, A\&A 458, 885-893 (2006).

[17] A. van der Meer, L. Kaper, T. di Salvo, M. Méndez, M. van der Klis, P. Barr, and N. R. Trams, XMM-Newton X-ray spectroscopy of the high-mass X-ray binary $4 U$ 1700-37 at low flux, A\&A 432, 999-1012 (2005).

[18] J. J. M. in 't Zand, T. E. Strohmayer, and A. Baykal, Dipping Activity in the X-Ray Pulsar $4 U$ 1907+09, ApJ 479, L47-L50 (1997).

[19] F. Haberl, N. E. White, and T. R. Kallman, An EXOSAT X-ray observation of one orbital cycle of 4U 1700-37/HD 153919, ApJ 343, 409-425 (1989).

[20] R. Staubert, I. Kreykenbohm, P. Kretschmar, M. Chernyakoca, K. Pottschmidt, S. BenllochGarcia, J. Wilms, A. Santangelo, A. Segreto, A. v. Kienlin, L. Sidoli, S. Larsson, and N. Westergaard, INTEGRAL/IBIS Observations of VELA X-1 in a Flaring State in proceedings of The INTEGRAL Universe, (Munich) (Battrick, ed.), ESA SP-552, October 2004, p. 259.

[21] R. Krivonos, N. Produit, I. Kreykenbohm, R. Staubert, A. von Kienlin, C. Winkler, and N. Gehrels, A very intense X-ray outburst of Vela X-1 detected with INTEGRAL, ATEL 211 (2003).

[22] P. R. den Hartog, W. Hermsen, L. M. Kuiper, J. J. M. in't Zand, C. Winkler, and A. Domingo, IGR J07597-3842 - A new INTEGRAL source with candidate counterparts in the radio, IR and $X$-ray bands, ATEL 261 (2004).

[23] S. Sazonov, E. Churazov, M. Revnivtsev, A. Vikhlinin, and R. Sunyaev, Identification of 8 INTEGRAL hard X-ray sources with Chandra, A\&A 444, L37-L40 (2005).

[24] F. Haberl, ROSAT soft X-ray observations of the supergiant X-ray binary Vela X-1, A\&A 288, 791 (1994).

[25] R. E. Taam and B. A. Fryxell, Numerical studies of asymmetric adiabatic accretion flow - The effect of velocity gradients, ApJ 339, 297-313 (1989).

[26] J. M. Blondin, T. R. Kallman, B. A. Fryxell, and R. E. Taam, Hydrodynamic simulations of stellar wind disruption by a compact X-ray source, ApJ 356, 591-608 (1990).

[27] T. Matsuda, N. Sekino, K. Sawada, E. Shima, M. Livio, U. Anzer, and G. Boerner, On the stability of wind accretion, A\&A 248, 301-314 (1991). 
[28] J. S. Benensohn, D. Q. Lamb, and R. E. Taam, Hydrodynamical Studies of Wind Accretion onto Compact Objects: Two-dimensional Calculations, ApJ 478, 723-733 (1997).

[29] L. Kaper, G. Hammerschlag-Hensberge, and J. T. van Loon, Observations of stellar winds in high-mass X-ray binaries: Evidence for a non-monotonic velocity structure, A\&A 279, 485-498 (1993).

[30] J.-C. Leyder, R. Walter, M. Lazos, N. Masetti, and N. Produit, Hard X-ray flares in IGR J08408-4503 unveil clumpy stellar winds, A\&A 465, L35-L38 (2007).

[31] A. Feldmeier, J. Puls, and A. W. A. Pauldrach, A possible origin for X-rays from O stars., A\&A 322, 878-895 (1997).

[32] R Walter and J Zurita-Heras, Probing clumpy stellar winds with a neutron star, A\&A 476, 335 (2007).

[33] M. C Runacres and S. P Owocki, A pseudo-planar, periodic-box formalism for modelling the outer evolution of structure in spherically expanding stellar winds, A\&A 429, 323 (2005).

[34] A. F. Illarionov and R. A. Sunyaev, Why the Number of Galactic X-ray Stars Is so Small?, A\&A 39, 185 (1975).

[35] Wei Cui, Evidence for "propeller" effects in X-ray pulsars GX 1+4 and GRO j1744-28, ApJ 482, L163 (1997).

[36] I. Kreykenbohm, W. Coburn, J. Wilms, P. Kretschmar, R. Staubert, W. A. Heindl, and R. E. Rothschild, Confirmation of two cyclotron lines in vela $x$-1, A\&A 395, 129-140 (2002).

[37] V. Sguera, E. J. Barlow, A. J. Bird, D. J. Clark, A. J. Dean, A. B. Hill, L. Moran, S. E. Shaw, D. R. Willis, A. Bazzano, P. Ubertini, and A. Malizia, INTEGRAL observations of recurrent fast X-ray transient sources, A\&A 444, 221-231 (2005).

[38] I. Negueruela, J. M. Torrejón, P. Reig, M. Ribó, and D. M. Smith, Supergiant Fast X-ray Transients and Other Wind Accretors in proceedings of A population explosion: the nature and evolution of X-ray binaries in diverse environments, (St. Petersburg, Florida) (R. M. Bandyopadhyay, ed.), AIP Conf. Proc. 1010, November 2008, p. 252.

[39] S. A. Grebenev and R. A. Sunyaev, The first observation of AX J1749.1-2733 in a bright $X$-ray state - Another fast transient revealed by INTEGRAL, Ast. Let. 33, 149-158 (2007).

[40] E. Bozzo, M. Falanga, and L. Stella, Are There Magnetars in High-Mass X-Ray Binaries? The Case of Supergiant Fast X-Ray Transients, ApJ 683, 1031-1044 (2008). 